VoL. 59 (1999) [187-201]

\title{
SYMMETRIC GEODESICS ON CONFORMAL COMPACTIFICATIONS OF EUCLIDEAN JORDAN ALGEBRAS
}

\author{
Sang Youl Lee, Yongdo lim and Chan-Young Park
}

\begin{abstract}
In this article we define symmetric geodesics on conformal compactifications of Euclidean Jordan algebras and classify symmetric geodesics for the Euclidean Jordan algebra of all $n \times n$ symmetric real matrices. Furthermore, we show that the closed geodesics for the Euclidean Jordan algebra of all $2 \times 2$ symmetric real matrices are realised as the torus knots in the Shilov boundary of a Lie ball.
\end{abstract}

\section{Introduction AND PRELIMINARIES}

A commutative algebra $V$ over field $\mathbb{R}$ or $\mathbb{C}$ with product $x y$ is said to be a Jordan algebra if for all elements $x, y$ in $V, x\left(x^{2} y\right)=x^{2}(x y)$. For $x \in V$, let $L(x)$ be the linear map of $V$ defined by $L(x) y=x y$, and let $P(x)=2 L(x)^{2}-L\left(x^{2}\right)$. A finite dimensional real Jordan algebra $V$ is called a Euclidean Jordan algebra if it admits an inner product $\langle x \mid y\rangle$ such that $\langle x y \mid z\rangle=\langle y \mid x z\rangle$. Let $V$ be a Euclidean Jordan algebra with identity $e$ and let $Q$ be the set of squares, and let $\Omega$ be the interior of $Q$. Then $\Omega$ is a self-dual cone and the group $G(\Omega):=\{g \in G L(V) \mid g \Omega=\Omega\}$ acts on it transitively. The tube domain $T_{\Omega}:=V+i \Omega$ associated with $\Omega$ is a symmetric tube domain which is biholomorphically isomorphic to a bounded symmetric domain via a Cayley transform. The Lie group $G\left(T_{\Omega}\right)$ of all biholomorphic automorphisms on the tube domain $T_{\Omega}$ can be described in the following way: an element in $G(\Omega)$ acts on the tube domain $T_{\Omega}$ by $g(z)=g(x)+i g(y), z=x+i y$. For $x \in V$, the translation by $x, t_{x}: z \mapsto z+x$ is a holomorphic automorphism of $T_{\Omega}$ and the group $N^{+}$of all real translations is an Abelian group isomorphic to the vector space $V$. The map $j: z \mapsto-z^{-1}$, the symmetry at $i e$, belongs to $G\left(T_{\Omega}\right)$. Let $\tilde{t}_{x}=j \circ t_{x} \circ j, N^{-}=j \circ N^{+} \circ j$. Then $G\left(T_{\Omega}\right)$ is generated by $N^{+}, G(\Omega)$ and $j[4]$.

Let $\mathfrak{n}^{ \pm}$be the Lie algebra of $N^{ \pm}$and let $\mathfrak{h}$ be the Lie algebra of $G(\Omega)$. Then the Lie algebra $\mathfrak{g}\left(T_{\Omega}\right)$ of $G\left(T_{\Omega}\right)$ can be identified with $\mathfrak{n}^{+}+\mathfrak{h}+\mathfrak{n}^{-}$in the following way:

\section{Received 27th May, 1998}

The work of the second author was partially supported by Korea Research Foundation, 1997. The work of the third author was partially supported by the Basic Science Research Institute Program Ministry of Education, 1997. Project No. 97-1409 and TGRC-KOSEF.

Copyright Clearance Centre, Inc. Serial-fee code: 0004-9729/99 \$A2.00+0.00. 
Let $g_{t}$ be a one-parameter subgroup of $G\left(T_{\Omega}\right)$. Then

$$
\tilde{X} f(z)=\left.\frac{d}{d t} f\left(g_{t}(z)\right)\right|_{t=0}, f \in \mathcal{C}^{1}\left(T_{\Omega}\right),
$$

defines a vector field on $T_{\Omega}$ and the set of vector fields obtained in this way is a real Lie subalgebra for the usual Lie bracket. We can write $\tilde{X} f(z)=D f(z)(X(z))$, where, for each $z, X(z)$ is a vector in $V_{\mathbf{C}}=V+i V$. Then the Lie algebra of $G\left(T_{\Omega}\right)$ is the set of vector fields of the form $X(z)=u+T z+P(z) v$, where $u, v \in V$ and $T \in \mathfrak{h}[4$, Theorem X.5.10]. Note that the vector field $X(z)=P(z) v$ corresponds to the one-parameter subgroup $\tilde{t}_{t v}$ in $N^{-}$[4]. A vector field $X$ in $\mathfrak{g}\left(T_{\Omega}\right), X(z)=u+T z+P(z) v$, can be identified with $(u, T, v) \in V \times \mathfrak{h} \times V$. Then

$$
\begin{aligned}
\mathfrak{n}^{+} & =\{(u, 0,0) \mid u \in V\} \cong V, \\
\mathfrak{h} & =\{(0, T, 0) \mid T \in \mathfrak{g}(\Omega)\}, \\
\mathfrak{n}^{-} & =\{(0,0, v) \mid v \in V\} \cong V .
\end{aligned}
$$

It is known [9] that $\mathfrak{g}\left(T_{\Omega}\right)$ is a symmetric algebra of Cayley type. Hence

$$
(X, h, Y) \in \mathrm{n}^{+} \times G(\Omega) \times \mathrm{n}^{-} \mapsto(\exp X) h(\exp Y) \in N^{+} G(\Omega) N^{-}
$$

is a diffeomorphism and $N^{+} G(\Omega) N^{-}$is a dense open subset in $G\left(T_{\Omega}\right)$. Set $P=G(\Omega) N^{-}$. Then $P$ is a maximal parabolic subgroup of $G\left(T_{\Omega}\right)$ and the homogeneous space $\mathcal{M}:=$ $G\left(T_{\Omega}\right) / P$ is a compact real manifold containing $V$ as an open dense subset, that is, a real conformal compactification of the Jordan algebra $V$. The embedding $V \rightarrow \mathcal{M}$ is given by $x \mapsto t_{x} P$. Furthermore, the set $N^{+} G(\Omega) N^{-}$can be characterised by the elements $g \in G\left(T_{\Omega}\right)$ such that $g \cdot 0 \in V$, where $\mathbf{0}$ is the base point $P$ corresponding to the zero vector in $V[3,7]$.

For $X \in \mathfrak{g}\left(T_{\Omega}\right)$, we assign $f(X) \in(0, \infty]$ as follows: if the solution $x(t)$ of the system $x^{\prime}(t)=X(x(t))$ with the initial value $x(0)=0$ stays in $V$ for all $t \in \mathbb{R}^{+}$, then $f(X)=\infty$. Otherwise, $f(X)$ is the first positive time reaching the boundary $\partial V \subset \mathcal{M}$. In this case the solution $x(t)$ is given by $\exp t X \cdot 0$. One way to evaluate $f(X)$ using the fact that $g \cdot 0 \in V$ if and only if $g \in N^{+} G(\Omega) N^{-}$is to decompose the exponential $\exp t X$ into $N^{+} G(\Omega) N^{-}$. Some explicit formulae for the factorisation of $\exp X$ for certain $X \in \mathfrak{q}:=\mathfrak{n}^{+}+\mathfrak{n}^{-}$were given in $[6,7,10]$.

Let $\operatorname{Con}_{G(\Omega)}(\mathfrak{q})$ be the set of closed, generating convex cones in $\mathfrak{q}$ which are invariant under the adjoint action of $G(\Omega)$. Then (see $[8,5]) \operatorname{Con}_{G(\Omega)}(\mathfrak{q})=\left\{C_{\mathfrak{p}},-C_{\mathrm{p}}, C_{\mathfrak{k}},-C_{\mathfrak{k}}\right\}$, where $C_{\mathfrak{p}}=\left\{(u, 0,-v) \in \mathfrak{g}\left(T_{\Omega}\right) \mid u, v \in \bar{\Omega}\right\}, C_{\mathfrak{l}}=\left\{(u, 0, v) \in \mathfrak{g}\left(T_{\Omega}\right) \mid u, v \in \bar{\Omega}\right\}$. It turns out $[8,9]$ that the wedge $\mathfrak{h}+C_{\mathfrak{p}}$ (respectively, $\mathfrak{h}-C_{\mathfrak{p}}$ ) is a Lie wedge of the compression semigroup of $\Omega$ (respectively, $-\Omega$ ) with a triple factorisation in $N^{+} G(\Omega) N^{-}$. Hence for $X \in\left(\mathfrak{h} \pm C_{\mathrm{p}}\right), f(X)=\infty$.

To complete a semisimple Jordan algebra $V$ of classical type to a symmetric space, Makarevič [11] used the term of geodesics in $V$ that originate at the zero point. In 
a Euclidean Jordan algebra $V$, these geodesics are eventually of the form $\alpha(t, a):=$ $\exp t X_{a} \cdot 0$, where $X_{a}=(a, 0, a) \in \mathfrak{q}$. Our main interest is in these geodesics and, in particular, in closed geodesics invariant for the symmetry $j$, which are said to be symmetric geodesics.

In this paper we shall confine ourselves to the Jordan algebra $V=\operatorname{Sym}(n, \mathbb{R})$ of $n \times n$ real symmetric matrices. An explicit expression of the exit time function $f$ on $\pm C_{\mathrm{e}}$ is given in Section 3. In Section 4, we classify symmetric geodesics and characterise some properties of these geodesics. In Section 5, we show that the closed geodesics for the Euclidean Jordan algebra $V=\operatorname{Sym}(2, \mathbb{R})$ of all $2 \times 2$ symmetric real matrices are realised as the torus knots in the Shilov boundary $\Sigma_{2}$ of a Lie ball.

\section{SyMPLECTIC GROUPS}

For a natural number $n, M_{n}(\mathbb{R})$ denotes the space of all $n$ by $n$ real matrices, and $A \in M_{n}(\mathbb{R})$ is symmetric (skew-symmetric) means that $A^{t}=A\left(A^{t}=-A\right)$. Let $\operatorname{Sym}(n, \mathbb{R})($ respectively $\operatorname{Skew}(n, \mathbb{R})$ ) be the space of all symmetric (respectively, skewsymmetric) $n$ by $n$ matrices. For a positive semi-definite symmetric matrix $A$, by $A^{1 / 2}$ we shall mean the square root of $A$. Let $A \in \operatorname{Sym}(n, \mathbb{R})$ have the spectral decomposition $A=\sum_{i=1}^{n} \lambda_{i} C_{i}$, where $\left\{C_{i}\right\}$ is a complete system of orthogonal projections. Then the spectral norm $|A|$ of $A$ is defined by $|A|=\max \left\{\left|\lambda_{1}\right|, \cdots,\left|\lambda_{n}\right|\right\}$.

Let $(\cdot \mid \cdot)$ be the skew-symmetric form on $\mathbb{R}^{2 n}$ defined by $(u \mid v)=\langle J u \mid v\rangle$ for $u, v \in$ $\mathbb{R}^{2 n}$, where $J=\left(\begin{array}{cc}0 & I \\ -I & 0\end{array}\right)$. Here, $I$ stands for the $n \times n$ identity matrix. The symplectic group $\mathrm{Sp}(2 n, \mathbb{R})$ on $\mathbb{R}^{2 n}$ is the Lie group of all invertible transformations $g=\left(\begin{array}{ll}A & B \\ C & D\end{array}\right)$ satisfying one of the following equivalent conditions:

(1) $g$ preserves $(\cdot \mid \cdot)$.

(2) $g^{t} J g=J$.

(3) $A^{t} C, B^{t} D$ are symmetric and $A^{t} D-C^{t} B=I$.

The Lie algebra of $\operatorname{Sp}(2 n, \mathbb{R})$ is given by

$$
\mathfrak{s p}(2 n, \mathbb{R})=\left\{\left(\begin{array}{cc}
X & Y \\
Z & -X^{t}
\end{array}\right) \mid X \in M_{n}(\mathbb{R}), Y, Z \in \operatorname{Sym}(n, \mathbb{R})\right\} .
$$

It has a Cartan decomposition $\mathfrak{s p}(2 n, \mathbb{R})=\mathfrak{p} \oplus \mathfrak{k}$, where

$$
\begin{aligned}
& \mathfrak{p}=\left\{\left(\begin{array}{cc}
X & Y \\
Y & -X
\end{array}\right) \mid X, Y \in \operatorname{Sym}(n, \mathbb{R})\right\}, \\
& \mathfrak{k}=\left\{\left(\begin{array}{cc}
X & Y \\
-Y & X
\end{array}\right) \mid X \in \operatorname{Skew}(n, \mathbb{R}), Y \in \operatorname{Sym}(n, \mathbb{R})\right\} .
\end{aligned}
$$


Let $\tau=\left(\begin{array}{cc}-I & 0 \\ 0 & I\end{array}\right) \in G L(2 n, \mathbb{R})$ and let $\tau(g)=\tau \cdot g \cdot \tau$ for $g \in \operatorname{Sp}(2 n, \mathbb{R})$. Then $\tau$ is an involution on $\operatorname{Sp}(2 n, \mathbb{R})$. The differential $\mathrm{d} \tau$ of $\tau$ at the identity is given by

$$
\mathrm{d} \tau\left(\begin{array}{cc}
X & Y \\
Z & -X^{t}
\end{array}\right)=\left(\begin{array}{cc}
X & -Y \\
-Z & -X^{t}
\end{array}\right)
$$

The Lie algebra $\mathfrak{s p}(2 n, \mathbb{R})$ can be decomposed as the +1-eigenspace $\mathfrak{h}$ and the -1 eigenspace $q$ of $d \tau$ :

$$
\mathfrak{s p}(2 n, \mathbb{R})=\mathfrak{h} \oplus \mathfrak{q}=\mathfrak{h} \oplus \mathfrak{n}^{+} \oplus \mathfrak{n}^{-}, \quad \mathfrak{q}=\mathfrak{n}^{+} \oplus \mathfrak{n}^{-}
$$

where

$$
\begin{aligned}
\mathfrak{n}^{+} & =\left\{\left(\begin{array}{ll}
0 & Y \\
0 & 0
\end{array}\right) \mid Y \in \operatorname{Sym}(n, \mathbb{R})\right\}, \\
\mathfrak{n}^{-} & =\left\{\left(\begin{array}{ll}
0 & 0 \\
Z & 0
\end{array}\right) \mid Z \in \operatorname{Sym}(n, \mathbb{R})\right\}, \\
\mathfrak{h} & =\left\{\left(\begin{array}{cc}
X & 0 \\
0 & -X^{t}
\end{array}\right) \mid X \in M_{n}(\mathbb{R})\right\} .
\end{aligned}
$$

Let $N^{ \pm}$be the Lie subgroup of $\operatorname{Sp}(2 n, \mathbb{R})$ corresponding $\mathrm{n}^{ \pm}$respectively. Then

$$
\begin{aligned}
& N^{+}=\left\{\left(\begin{array}{ll}
I & A \\
0 & I
\end{array}\right) \mid A \in \operatorname{Sym}(n, \mathbb{R})\right\}=\exp \mathfrak{n}^{+}, \\
& N^{-}=\left\{\left(\begin{array}{ll}
I & 0 \\
A & I
\end{array}\right) \mid A \in \operatorname{Sym}(n, \mathbb{R})\right\}=\exp \mathfrak{n}^{-} .
\end{aligned}
$$

Let

$$
H=\left\{\left(\begin{array}{cc}
A & 0 \\
0 & \left(A^{-1}\right)^{t}
\end{array}\right) \mid A \in G L(n, \mathbb{R})\right\} .
$$

Note that $H=\{g \in \operatorname{Sp}(2 n, \mathbb{R}) \mid \tau(g)=g\}$.

Lemma 2.1. An element $g=\left(\begin{array}{ll}A & B \\ C & D\end{array}\right) \in \mathrm{Sp}(2 n, \mathbb{R})$ can be written (uniquely) as a triple product in $N^{+} H N^{-}$if and only if $D$ is invertible. In this case the (unique) factorisation is given by

$$
\left(\begin{array}{ll}
A & B \\
C & D
\end{array}\right)=\left(\begin{array}{cc}
I & B D^{-1} \\
0 & I
\end{array}\right)\left(\begin{array}{cc}
\left(D^{-1}\right)^{t} & A \\
0 & D
\end{array}\right)\left(\begin{array}{cc}
I & 0 \\
D^{-1} C & I
\end{array}\right)
$$

ProOF: See [9]. 
TheOREM 2.2. Let $P=H N^{-}$. Then $P$ is a closed subgroup of $G:=\operatorname{Sp}(2 n, \mathbb{R})$ and the homogeneous space $\mathcal{M}:=G / P$ is a compact real manifold with $V:=\operatorname{Sym}(n, \mathbb{R})$ as an open dense subset. The embedding of $V$ into $\mathcal{M}$ is given by

$$
X \in V \mapsto\left(\begin{array}{cc}
I & X \\
0 & I
\end{array}\right) \cdot P \in \mathcal{M}
$$

Furthermore, for $g=\left(\begin{array}{ll}A & B \\ C & D\end{array}\right) \in \mathrm{Sp}(2 n, \mathbb{R})$ and $X \in V$ with $g \cdot X \in V$, we have

$$
g \cdot X=(A X+B)(C X+D)^{-1} .
$$

Proor: This follours from the gonoral thoory of conformal compantificatione of Jor dan algebras $[1,2,3]$.

\section{3. $\operatorname{Ad}(H)$-INVARIANT Cones}

Let

$$
C_{\mathfrak{k}}=\left\{\left(\begin{array}{cc}
0 & A \\
-B & 0
\end{array}\right) \mid A, B \geqslant 0\right\}, \quad C_{\mathfrak{p}}=\left\{\left(\begin{array}{cc}
0 & A \\
B & 0
\end{array}\right) \mid A, B \geqslant 0\right\} .
$$

Then $C_{\mathfrak{k}},-C_{\mathfrak{k}}, C_{\mathfrak{p}},-C_{\mathfrak{p}}$ are pointed, generating $\operatorname{Ad}(H)$-invariant convex cones in $\mathfrak{q}$.

Let $A \in V=\operatorname{Sym}(n, \mathbb{R})$ have the spectral decomposition $A=\sum_{i=1}^{n} \lambda_{i} C_{i}$. If $g$ is a real valued function on an interval containing all eigenvalues $\lambda_{i}$, then we shall define an element $g(A) \in V$ by the formula $g(A)=\sum_{i=1}^{n} g\left(\lambda_{i}\right) C_{i}$. For instance, $\cos A, \sin A$ are defined in this way.

Lemma 3.1. Let $A \in V$ with the spectral decomposition $A=\sum_{i=1}^{n} \lambda_{i} C_{i}$. Then $\cos (t A)$ is singular if and only if

$$
t=\frac{\pi(2 k+1)}{2 \lambda_{i}}
$$

for some $i$ and for some $k \in \mathbb{Z}$.

Proof: This follows from the fact that $\cos (t A)=\sum_{i=1}^{n}\left(\cos t \lambda_{i}\right) C_{i}$ is invertible if and only if $\cos t \lambda_{i} \neq 0$ for all $i$.

For $A \in M_{n}(\mathbb{R})$, we let

$$
\begin{aligned}
& c(A)=\sum_{k=0}^{\infty} \frac{A^{k}}{(2 k) !} \\
& s(A)=\sum_{k=0}^{\infty} \frac{A^{k}}{(2 k+1) !} .
\end{aligned}
$$


Proposition 3.2. Let $A, B \in V$. Then

$$
\exp \left(\begin{array}{cc}
0 & t A \\
-t B & 0
\end{array}\right)=\left(\begin{array}{cc}
c\left(-t^{2} A B\right) & t A s\left(-t^{2} B A\right) \\
-t B s\left(-t^{2} A B\right) & c\left(-t^{2} B A\right)
\end{array}\right)
$$

In particular,

$$
\exp \left(\begin{array}{cc}
0 & t A \\
-t A & 0
\end{array}\right)=\left(\begin{array}{cc}
\cos (t A) & \sin (t A) \\
-\sin (t A) & \cos (t A)
\end{array}\right)
$$

Moreover, if $A$ and $B$ are positive definite matrices, then $\exp \left(\begin{array}{cc}0 & t A \\ -t B & 0\end{array}\right)$ equals

$$
\left(\begin{array}{cc}
B^{-1 / 2}\left[\cos t\left(B^{1 / 2} A B^{1 / 2}\right)^{1 / 2}\right] B^{1 / 2} & B^{-1 / 2}\left(B^{1 / 2} A B^{1 / 2}\right)^{1 / 2}\left[\sin t\left(B^{1 / 2} A B^{1 / 2}\right)^{1 / 2}\right] B^{-1 / 2} \\
-B^{1 / 2}\left(B^{1 / 2} A B^{1 / 2}\right)^{-1 / 2}\left[\sin t\left(B^{1 / 2} A B^{1 / 2}\right)^{1 / 2}\right] B^{1 / 2} & B^{1 / 2}\left[\cos t\left(B^{1 / 2} A B^{1 / 2}\right)^{1 / 2}\right] B^{-1 / 2}
\end{array}\right) .
$$

Proof: This follows from a direct matrix computation.

Corollary 3.3. Let $A=\sum_{i=1}^{n} \lambda_{i} C_{i} \in V$ be the spectral decomposition of $A$. Then

$$
\exp \left(\begin{array}{cc}
0 & t A \\
-t A & 0
\end{array}\right) \cdot 0 \in \partial V \Longleftrightarrow t=\frac{\pi(2 k+1)}{2 \lambda_{i}}
$$

for some $i$ and for some $k \in \mathbb{Z}$.

Proof: This follows from Lemma 2.1, Lemma 3.1, and Proposition 3.2.

Corollary 3.4. Let $A, B \geqslant 0$, (that is, $A$ and $B$ are positive semi-definite matrices). Let $\sum_{i=1}^{n} \lambda_{i} C_{i}$ be the spectral decomposition of $B^{1 / 2} A B^{1 / 2}$. Then

$$
\exp \left(\begin{array}{cc}
0 & t A \\
-t B & 0
\end{array}\right) \cdot 0 \in \partial V \Longleftrightarrow t=\frac{\pi(2 k+1)}{2 \sqrt{\lambda}_{i}}
$$

for some $i$ and for some $k \in \mathbb{Z}$.

Proof: First, we show that for $A, B \geqslant 0$, Det $c(-B A)=\operatorname{Det} \cos \left(B^{1 / 2} A B^{1 / 2}\right)^{1 / 2}$, where Det is the determinant function. Choose $A_{n}, B_{n}>0$ such that $A_{n} \rightarrow A$ and $B_{n} \rightarrow B$. Then

$$
c\left(-B_{n} A_{n}\right)=B_{n}^{1 / 2}\left[\cos \left(B_{n}^{1 / 2} A_{n} B_{n}^{1 / 2}\right)^{1 / 2}\right] B_{n}^{-1 / 2} .
$$

Thus

$$
\text { Det } \begin{aligned}
c(-B A) & =\lim _{n \rightarrow \infty} \operatorname{Det} c\left(-B_{n} A_{n}\right) \\
& =\lim _{n \rightarrow \infty} \operatorname{Det} B_{n}^{1 / 2}\left[\cos \left(B_{n}^{1 / 2} A_{n} B_{n}^{1 / 2}\right)^{1 / 2}\right] B_{n}^{-1 / 2} \\
& =\lim _{n \rightarrow \infty} \operatorname{Det} \cos \left(B_{n}^{1 / 2} A_{n} B_{n}^{1 / 2}\right)^{1 / 2} \\
& =\operatorname{Det} \cos \left(B^{1 / 2} A B^{1 / 2}\right)^{1 / 2}
\end{aligned}
$$


Now by Lemma 2.1, Lemma 3.1, and Proposition 3.2,

$$
\exp \left(\begin{array}{cc}
0 & t A \\
-t B & 0
\end{array}\right) \cdot 0 \in \partial V
$$

if and only if $c\left(-t^{2} B A\right)$ is singular if and only if $\cos t\left(B^{1 / 2} A B^{1 / 2}\right)^{1 / 2}$ is singular if and only if $t=(\pi(2 k+1)) /\left(2 \sqrt{\lambda_{i}}\right)$ for some $i$ and for some $k \in \mathbb{Z}$.

THEOREM 3.5. The exit function $f$ is continuous on $C_{\mathrm{k}}$. In particular,

$$
f^{-1}(\infty) \cap C_{\mathrm{e}}=\left\{\left(\begin{array}{cc}
0 & A \\
-B & 0
\end{array}\right) \in C_{\mathrm{l}} \mid B^{1 / 2} A B^{1 / 2}=0\right\} .
$$

Proof: By Corollary $3.4, f\left(\begin{array}{cc}0 & A \\ -B & 0\end{array}\right)=\pi /\left(2\left|B^{1 / 2} A B^{1 / 2}\right|^{1 / 2}\right)$.

REMARK 3.6. Note that for $g=\left(\begin{array}{ll}A & B \\ C & D\end{array}\right) \in \mathrm{Sp}(2 n, \mathbb{R}), g^{-1}=\left(\begin{array}{cc}D^{t} & -B^{t} \\ -C^{t} & A^{t}\end{array}\right)$. Therefore if $A, B \geqslant 0$, then

$$
\begin{aligned}
\exp \left(\begin{array}{cc}
0 & -t A \\
t B & 0
\end{array}\right) & =\exp \left(\begin{array}{cc}
0 & t A \\
-t B & 0
\end{array}\right)^{-1} \\
& =\left(\begin{array}{cc}
c\left(-t^{2} B A\right)^{t} & -t\left(A s\left(-t^{2} B A\right)\right)^{t} \\
t\left(B s\left(-t^{2} A B\right)\right)^{t} & c\left(-t^{2} A B\right)^{t}
\end{array}\right)
\end{aligned}
$$

Since $c\left(-t^{2} A B\right)^{t}=c\left(-t^{2} B A\right)$, the map $f$ is given on $-C_{\mathfrak{k}}$ by

$$
f\left(\begin{array}{cc}
0 & -A \\
B & 0
\end{array}\right)=\frac{\pi}{2\left|A^{1 / 2} B A^{1 / 2}\right|^{1 / 2}} .
$$

Hence $f$ is continuous on $-C_{\mathbf{k}}$.

\section{Geodesics on $\mathcal{M}$}

Let $A \in V=\operatorname{Sym}(n, \mathbb{R})$. Set $X_{A}:=\left(\begin{array}{cc}0 & A \\ -A & 0\end{array}\right) \in \mathfrak{k}$. Then it is known [11] that a geodesic in $V$ originating at the origin 0 with direction $A$ is of the form

$$
\alpha(t, A):=\exp t X_{A} \cdot \mathbf{0}=\left(\begin{array}{cc}
\cos t A & \sin t A \\
-\sin t A & \cos t A
\end{array}\right) \cdot 0 .
$$

Proposition 4.1. Let $A \in V$.

(1) If $\sin t A=0$ (respectively, $\cos t A=0)$ then $\cos t A($ respectively, $\sin t A)$ is invertible. 
(2) The geodesic $\alpha(t, A)$ touches the boundary of $V$ at time $t_{0}$ if and only if $\cos t_{0} A$ is singular. In particular, if $\cos t A$ is invertible, then $\alpha(t, A)=$ $\tan t A$.

(3) The geodesic $\alpha(t, A)$ is closed if and only if $\alpha\left(t_{0}, A\right)=0$ for some $t_{0} \neq 0$ if and only if $\sin t_{0} A=0$ for some $t_{0} \neq 0$.

(4) For the geodesic $\alpha(t, A), t=\pi /(2|A|)$ is the first positive time it touches the boundary of $V$.

(5) $\alpha\left(t, t^{\prime} A\right)=\alpha\left(t t^{\prime}, A\right)$ for any $t, t^{\prime} \in \mathbb{R}$.

PROOF: (1) and (5) are obvious. (2) follows from Lemma 2.1 and Proposition 3.2. By the definition of closed geodesic, $\alpha(t, A)$ is closed if and only if $\alpha\left(t_{0}, A\right)=0$ for some $t_{0} \neq 0$. By Lemma 2.1 and Proposition 3.2, this is equivalent to the condition $\sin t_{0} A=0$. (4) follows from Corollary 3.3.

The period of a non-constant closed geodesic $\alpha(t, A)$ is the smallest positive real number $t_{0}$ satisfying $\alpha\left(t_{0}, A\right)=\mathbf{0}$. Set

$$
E_{c}=\left\{r\left(p_{1}, \cdots, p_{n}\right) \in \mathbb{R}^{n} \mid r \geqslant 0, p_{i}: \text { integer }\right\} .
$$

In this setting, we always assume that the integers $p_{i}$ have no common divisors.

Theorem 4.2. Let $A=\sum_{i=1}^{n} \lambda_{i} C_{i}$ be the spectral decomposition of $A$. Then $\alpha(t, A)$ is a closed geodesic if and only if $\left(\lambda_{1}, \cdots, \lambda_{n}\right) \in E_{c}$. If $A \neq 0$ and $\left(\lambda_{1}, \cdots, \lambda_{n}\right)=$ $r\left(p_{1}, \cdots, p_{n}\right) \in E_{c}$, then $\pi / r$ is the period of $\alpha(t, A)$.

Proof: Suppose that $\alpha(t, A)$ is a closed geodesic. If $A=0$, then all $\lambda_{i}$ are zero and hence $\left(\lambda_{1}, \cdots, \lambda_{n}\right)=0(1, \cdots, 1) \in E_{c}$. Suppose that $A \neq 0$. Let $\lambda_{k}$ be the first non-zero eigenvalue of $A$. By Proposition 4.1(3), there exists $t_{0} \neq 0$ such that $\sin t_{0} A=0$. This implies that $\sin t_{0} \lambda_{i}=0$ for all $i=1,2, \cdots, n$. From this, we conclude that $\lambda_{i} / \lambda_{k} \in \mathbb{Q}$ (the field of rational numbers) for all $i=1,2, \cdots, n$. Then for $i=k+1, \cdots, n$, there exists $r_{i} \in$ $\mathbb{Q}$ such that $\lambda_{i}=\lambda_{k} r_{i}$. By multiplying by the least common multiple of the denominators of the $r_{i}$, we can rewrite $\left(\lambda_{1}, \cdots, \lambda_{k}, \cdots, \lambda_{n}\right)$ as $r\left(0, \cdots, 0, p_{k}, p_{k+1}, \cdots, p_{n}\right) \in E_{c}$ for some $p_{i} \in \mathbb{Z}, r>0$.

Conversely, suppose that $\left(\lambda_{1}, \cdots, \lambda_{n}\right)=r\left(p_{1}, \cdots, p_{n}\right) \in E_{c}$. If $r=0$, then $\alpha(t, A)=$ 0 is constant and hence is a closed geodesic. If $r \neq 0$, then $\sin (\pi / r) \lambda_{i}=\sin p_{i} \pi=0$ for all $i$. This implies that $\sin (\pi / r) A=0$ and hence $\alpha(t, A)$ is a closed geodesic.

Finally suppose that $A \neq 0$ and $\alpha\left(t_{0}, A\right)=0$ for some $t_{0}>0$. Since $A \neq 0, r \neq 0$ and $p_{k} \neq 0$ for some $k$. Hence $\sin t_{0} \lambda_{k}=\sin t_{0} r p_{k}=0$ implies that $t_{0}=\left(m \pi / r p_{k}\right)$ for some integer $m$. If $p_{k}= \pm 1$, then $t_{0} \geqslant \pi / r$. Suppose $p_{k} \neq \pm 1$. Since $\left\{p_{i}\right\}$ have no common divisors, there exists $j \neq k$ such that $p_{j} \neq 0$ and $p_{k}$ and $p_{j}$ are relatively prime. Since $\sin t_{0} \lambda_{j}=\sin t_{0} r p_{j}=0, t_{0}=\left(m^{\prime} \pi / r p_{j}\right)$ for some integer $m^{\prime}$. Therefore 
$t_{0}=\left(m \pi / r p_{k}\right)=\left(m^{\prime} \pi / r p_{j}\right)$. Since $p_{k}$ and $p_{j}$ are relatively prime, $p_{k}$ must divide $m$ and hence $t_{0} \geqslant \pi / r$.

Set $j=\left(\begin{array}{cc}0 & -I \\ I & 0\end{array}\right) \in \operatorname{Sp}(2 n, \mathbb{R})$. Then $j$ is an involution on $\mathcal{M}$ and for an invertible element $A=\sum_{i=1}^{n} \lambda_{i} C_{i}, j \cdot A=-A^{-1}$.

Proposition 4.3. Let $A \in V$. Then the following conditions are equivalent:

(1) For any $t \in \mathbb{R}$, there exists $t^{\prime}$ (depends on $t$ ) such that $j \cdot \alpha(t, A)=\alpha\left(t^{\prime}, A\right)$.

(2) There exists a non-zero real number $t_{0} \in \mathbb{R}$ such that $j \cdot \alpha(t, A)=\alpha\left(t+t_{0}, A\right)$ for a.ll $t \in \mathbb{R}$.

(3) There exists a non-zero real number $t_{0}$ such that $\cos t_{0} A=0$.

In particular, if a geodesic $\alpha(t, A)$ satisfies one of these conditions, then $A$ is invertible.

ProOF: By Lemma 2.1, we have

$$
\left(\exp t X_{A}\right) j=\left(\begin{array}{cc}
\sin t A & -\cos t A \\
\cos t A & \sin t A
\end{array}\right) \in P \text { if and only if } \cos t A=0 .
$$

(1) implies (3) : Let $t$ be a non-zero real number. Then there is a real number $t^{\prime}$ such that $j \cdot \alpha(t, A)=\alpha\left(t^{\prime}, A\right)$. That is, $j \exp t X_{A} \cdot 0=\exp t^{\prime} X_{A} \cdot 0$. Since $j X_{A} j^{-1}=X_{A}$, we have

$$
\begin{aligned}
0 & =\exp \left(-t^{\prime} X_{A}\right) j \exp t X_{A} \cdot \mathbf{0} \\
& =\exp \left(-t^{\prime} X_{A}\right) j \exp t X_{A} j^{-1} j \cdot \mathbf{0} \\
& =\exp \left(-t^{\prime} X_{A}\right) \exp t X_{A} j \cdot \mathbf{0} .
\end{aligned}
$$

Hence $\left(\exp \left(t-t^{\prime}\right) X_{A}\right) j \in P$. Set $t_{0}=t-t^{\prime}$. Then $t_{0} \neq 0$ since $j$ is not in $P$. Therefore by (4.1) $\cos t_{0} A=0$.

(3) implies (2): Suppose that $\cos t_{0} A=0$ for some non-zero $t_{0} \in \mathbb{R}$. Then by (4.1), $\left(\exp t_{0} X_{A}\right) j \cdot 0=0$ and hence for any $t \in \mathbb{R}$,

$$
\begin{aligned}
j\left(\exp t X_{A}\right) \cdot \mathbf{0} & =j\left(\exp \left(t+t_{0}\right) X_{A}\right)\left(\exp -t_{0} X_{A}\right) \cdot \mathbf{0} \\
& =j\left(\exp \left(t+t_{0}\right) X_{A}\right) j \cdot \mathbf{0} \\
& =j\left(\exp \left(t+t_{0}\right) X_{A}\right) j^{-1} \cdot \mathbf{0} \\
& =\left(\exp \left(t+t_{0}\right) X_{A}\right) \cdot \mathbf{0} .
\end{aligned}
$$

Therefore $j \cdot \alpha(t, A)=\alpha\left(t+t_{0}, A\right)$.

(2) implies (1): Trivial.

Now suppose $\cos t_{0} A=0$ for some $t_{0} \neq 0$. Then $\cos t_{0} \lambda_{i}=0$ and hence $\lambda_{i} \neq 0$ for all $i$. Therefore $A$ is invertible. 
Definition. A closed geodesic $\alpha(t, A)$ is said to be symmetric if it satisfies one of the conditions of Proposition 4.3.

Set $\mathbb{R}^{*}=\mathbb{R} \backslash\{0\}$. Let $E_{s}$ be the subset of $\left(\mathbb{R}^{*}\right)^{n}$

$$
E_{s}=\left\{r\left(p_{1}, p_{2}, \cdots, p_{n}\right) \mid r>0, p_{i}: \text { odd integer }\right\}
$$

Then $E_{s}$ is a subgroup of $\left(\mathbb{R}^{*}\right)^{n}$ under the usual multiplication.

TheOREM 4.4. Let $A=\sum_{i=1}^{n} \lambda_{i} C_{i} \in V$ be the spectral decomposition of $A$. Then $\alpha(t, A)$ is a symmetric geodesic if and only if $\left(\lambda_{1}, \cdots, \lambda_{n}\right) \in E_{s}$.

Proof: If $\left(\lambda_{1}, \cdots, \lambda_{n}\right)=r\left(p_{1}, \cdots, p_{n}\right) \in E_{s}$, then $\cos (\pi / 2 r) A=0$. Hence $\alpha(t, A)$ is symmetric. Conversely, suppose that $\alpha(t, A)$ is symmetric. Then by Propositions 4.1 and $4.3, \cos t A=0$ and $\sin t^{\prime} A=0$ for some $t \neq 0$ and $t^{\prime} \in \mathbb{R}$. By Theorem 4.2, $\left(\lambda_{1}, \cdots, \lambda_{n}\right)=r\left(p_{1}, \cdots, p_{n}\right)$ for some integers $p_{i}$ which have no common divisors. Since $A$ is invertible (by Proposition 4.3), $r$ and the $p_{i}$ 's are all non-zero. Note that $\cos t A=0$ implies that $p_{i}-p_{j}$ is even for all $i, j=1, \cdots, n$. If $p_{1}$ is even, then all the $p_{i}$ 's are even. But this is impossible since they have no common divisors. Thus $p_{1}$ is odd and hence all the $p_{i}$ 's are odd integers.

Corollary 4.5. Let $\alpha(t, A)$ be a symmetric geodesic. Let $A=\sum_{i=1}^{n} r p_{i} C_{i}$ be the spectral decomposition of $A$. Then $j \cdot \alpha(t, A)=\alpha(t+(\pi / 2 r), A)$. In particular, $j \cdot 0=\alpha(\pi / 2 r, A)$.

ProOF: Let $\alpha(t, A)$ be a symmetric geodesic. Note that $r>0$ and the $p_{i}$ 's are all odd numbers (they have no common divisors). Set $A^{\prime}=(1 / r) A$. For $i=1, \cdots, n$, $\tan (t+\pi / 2) p_{i}=-\cot \left(t p_{i}\right)$ whenever it is defined. This implies that for every $t>0$ with $j \cdot \alpha\left(t, A^{\prime}\right) \in V$,

$$
j \cdot \alpha\left(t, A^{\prime}\right)=-\cot t A^{\prime}=\tan \left(t+\frac{\pi}{2}\right) A^{\prime}=\alpha\left(+t+\frac{\pi}{2}, A^{\prime}\right) .
$$

Because $V$ is dense in $\mathcal{M}$, the equality holds for all $t$. By Proposition 4.1, we have

$$
j \cdot \alpha(t, A)=j \cdot \alpha\left(t r, A^{\prime}\right)=\alpha\left(t r+\frac{\pi}{2}, A^{\prime}\right)=\alpha\left(t+\frac{\pi}{2 r}, A\right) .
$$

Corollary 4.6. Let $\alpha(t, A)$ be a symmetric geodesic. If $\alpha\left(t_{0}, A\right) \in \partial V$, then $j \cdot \alpha\left(t_{0}, A\right) \in V$.

Proof: Let $\sum_{i=1}^{n} r p_{i} C_{i}$ be the spectral decomposition of $A$. Since $\alpha\left(t_{0}, A\right) \in \partial V$, $\cos t_{0} A$ is singular. Hence $t_{0}=\left(k / 2 r p_{i}\right) \pi$ for some $i$ and an odd integer $k$. Since, by Corollary $4.5, j \cdot \alpha\left(t_{0}, A\right)=\alpha\left(t_{0}+(\pi / 2 r), A\right)$, we claim that $\cos \left(t_{0}+(\pi / 2 r)\right) r p_{j} \neq 0$ for 
all $j=1, \cdots, n$. It then follows from $\cos \left(t_{0}+(\pi / 2 r)\right) r p_{j}=\cos \left(\left(k p_{j} / 2 p_{i}\right)+\left(p_{j} / 2\right) \pi\right)=$ $-\sin \left(\left(k p_{j} / 2 p_{i}\right) \pi\right) \neq 0$ because $p_{i}, p_{j}$, and $k$ are odd integers. This ends the proof.

REMARKs 4.7. (1) By Corollary 4.6, boundary points in a symmetric geodesic curve have to move into $V$ by the symmetry $j$. This property distinguishes symmetric geodesics among closed geodesics. Let $\alpha(t, A)$ be a non-constant closed geodesic. Then it touches the boundary $\partial V$ finitely many times (Proposition 4.1 (4)). Suppose that $\alpha(t, A)$ is nonsymmetric. Then an eigenvalue $\lambda_{k}$ of $A$ is $r p_{k}$ for some even integer $p_{k}$. Here, $\pi / r$ is the period of the given geodesic curve. Since $\left\{p_{i}:=\lambda_{i} / r\right\}$ have no common divisors, $p_{j}$ is odd for some $j$. This implies that $\sin \left((\pi / 2 r) r p_{k}\right)=0$ and $\cos \left((\pi / 2 r) r p_{j}\right)=0$. Therefore, $\alpha((\pi / 2 r), A) \in \partial V$ and $j \cdot \alpha((\pi / 2 r), A) \in \partial V$.

(2) Lot $\left.a_{(}^{\prime}, A\right)$ bo a summetris gendesic with the snertral dernmnnsition $A=$ $\sum_{i=1}^{n} r p_{i} C_{i}$. Let $A^{\prime}=(1 / r) A$. If $\alpha\left(t_{0}, A^{\prime}\right) \in \partial V$, then $t_{0}$ is of the form $\left(k / 2 p_{i}\right) \pi$ for some $i$ and some odd integer $k$. Since $2 p_{i}-k$ is an odd number, $\cos \left(\pi-t_{0}\right) p_{i}=$ $\left.\cos \left(\left(2 p_{i}-k\right) / 2\right)\right) \pi=0$. Hence $\alpha\left(\pi-t_{0}, A^{\prime}\right) \in \partial V$. If $0<t_{0}<\pi / 2$, then $\pi / 2<\pi-t_{0}=\left(1-\left(k / 2 p_{i}\right)\right) \pi<\pi$. Similarly if $\pi / 2<t_{0}<\pi$, then $0<\pi-t_{0}<\pi / 2$. This implies the number of boundary points in the geodesic curve $\alpha\left(t, A^{\prime}\right)$ is odd, centred at the time $\pi / 2$. This holds for $A$ since $\alpha\left(t, A^{\prime}\right)=\alpha((t / r), A)$. Moreover $\pi / r-\pi /(2|A|)$ is the last time of touching the boundary since $\pi /(2|A|)$ is the first one. In the case $n=2$, one may easily show that the number of boundary points in the symmetric geodesic curve $\alpha(t, A)$ is $p_{1}+p_{2}-1$.

\section{Closed GeOdesics For $\operatorname{Sym}(2, \mathbb{R})$}

Let $V=\operatorname{Sym}(2, \mathbb{R})$ and let $\Omega$ be the symmetric cone of positive definite $2 \times 2$ symmetric real matrices. Then the tube domain $T_{\Omega}:=V+i \Omega$ can be realised as a bounded symmetric domain $\mathcal{D}$ in the complex plane $V^{\mathbb{C}}:=V+i V$ as follows: we define

$$
\begin{aligned}
& D(p)=\left\{Z \in V^{\mathbb{C}} \mid Z+i I \in G L(2, \mathbb{C})\right\}, \\
& D(c)=\left\{W \in V^{\mathbb{C}} \mid I-W \in G L(2, \mathbb{C})\right\}
\end{aligned}
$$

and for all $Z \in D(p), W \in D(c)$,

$$
\begin{aligned}
p(Z) & =(Z-i I)(Z+i I)^{-1} \\
c(W) & =i(I+W)(I-W)^{-1} .
\end{aligned}
$$

Then the map $p$ is a holomorphic bijection of $D(p)$ onto $D(c)$ and $c$, called the Cayley transform, is its inverse. The closure of $T_{\Omega}$ in $V^{\mathbf{C}}$ is contained in $D(p)$. The image $\mathcal{D}:=p\left(T_{\Omega}\right)$ of $p$ is known as a bounded symmetric domain, called a Lie ball (open unit ball with respect to the spectral norm) [4]. We define $\Sigma_{2}$ as the set of invertible elements 
in $V^{\mathbb{C}}$ such that $Z^{-1}=\bar{Z}$. It is known [4] that $\Sigma_{2}$ is the Shilov boundary of $\mathcal{D}$, which is a compact connected 3-dimensional manifold, and is exactly equal to $\overline{p(V)}$.

Let $\mathbf{c}=\left\{C_{i}\right\}_{i=1}^{2}$ be a complete system of orthogonal projections (Jordan frame) and let $V(\mathrm{c})$ be the subspace of $V$ generated by $C_{i}^{\prime} s$. Then for $A=\sum_{i=1}^{2} \lambda_{i} C_{i}$,

$$
p(A)=\sum_{i=1}^{2} \frac{\lambda_{i}-i}{\lambda_{i}+i} C_{i}
$$

Since $\left(\lambda_{i}-i\right) /\left(\lambda_{i}+i\right) \in S^{1}$, the unit circle in $\mathbb{C}$, for $i=1,2$, we conclude that $\overline{p(V(\mathbf{c}))}$ is diffeomorphic to the torus $T^{2}=S^{1} \times S^{1}$ embedded in $\Sigma_{2}$.

For a geodesic curve $\alpha(t, A)$ in $\mathcal{M}$, we let $\widehat{\alpha}(t, A):=p(\alpha(t, A))$ be the corresponding geodesic on $\Sigma_{2}$. From (5.2), we have the following.

Proposition 5.1. Let $A=\sum_{i=1}^{2} \lambda_{i} C_{i}$ be the spectral decomposition of $A$. Then

$$
\widehat{\alpha}(t, A)=\sum_{i=1}^{2} e^{i\left(\pi+2 \lambda_{i} t\right)} C_{i} .
$$

Proof: For $t>0$ with $\alpha(t, A) \in V, p(\alpha(t, A))=\sum_{i=1}^{2}\left(\left(\tan \lambda_{i} t-i\right) /\left(\tan \lambda_{i} t+i\right)\right) C_{i}$. The result then follows from

$$
\frac{\tan \lambda_{i} t-i}{\tan \lambda_{i} t+i}=\sin ^{2} \lambda_{i} t \cos ^{2} \lambda_{i} t-2 i \sin \lambda_{i} t \cos \lambda_{i} t=e^{i\left(\pi+2 \lambda_{i} t\right)} .
$$

A torus knot is a simple closed curve embedded on the standard torus $T^{2}=S^{1} \times S^{1}$ in $\mathbb{C}^{2}$. Any point of $T^{2}$ has coordinates $\left(e^{i \psi}, e^{i \phi}\right)$. We have the standard meridian-longitude generator system of $H_{1}\left(T^{2}\right) \cong \mathbb{Z} \oplus \mathbb{Z}$ :

$$
\begin{aligned}
m: e^{i \theta} \mapsto\left(e^{i \theta}, 1\right), & \text { meridian, } \\
\ell: e^{i \theta} \mapsto\left(1, e^{i \theta}\right), & \text { longitude. }
\end{aligned}
$$

A torus knot is said to be of type $(p, q)$, denoted by $T(p, q)$, if it is homologous to $p m+q \ell$ on $T^{2}$ and $p$ and $q$ are coprime integers. A torus knot $T(p, q)$ is trivial if and only if either $p= \pm 1$ or $q= \pm 1$. Two nontrivial torus knots $T(p, q)$ and $T\left(p^{\prime}, q^{\prime}\right)$ are equivalent if and only if $\left(p^{\prime}, q^{\prime}\right)$ is equal to one of $(p, q),(q, p),(-p, q)$, or $(-p,-q)$ [12].

THEOREM 5.2. Let $A=\sum_{i=1}^{2} r p_{i} C_{i}\left(r \geqslant 0, p_{i} \in \mathbb{Z}\right)$ be the spectral decomposition of $A \in \operatorname{Sym}(2, \mathbb{R})$ and let $\alpha(t, A)$ be the corresponding closed geodesic in $\mathcal{M}$. Then $\widehat{\alpha}(t, A)(0 \leqslant t \leqslant \pi / r)$ is the torus knot of type $\left(\left|p_{1}\right|,\left|p_{2}\right|\right)$. 
Proof: Let $K=\left\{\widehat{\alpha}(t, A) \in V=\Sigma_{2} \mid 0 \leqslant t \leqslant \pi / r\right\}$. By Proposition 5.1, $K$ is a simple closed curve on the standard torus $T^{2}=S^{1} \times S^{1}$ embedded in $\mathbb{C}^{2}$. Since $\alpha(t, A)$ is a closed geodesic, $p_{1}$ and $p_{2}$ are relatively prime integers. Now

$$
\begin{aligned}
K & =\left\{e^{i \pi}\left(e^{i 2 r p_{1} t}, e^{i 2 r p_{2} t}\right) \in T^{2} \mid 0 \leqslant t \leqslant \pi / r\right\} \\
& =\left\{\left(-e^{i p_{1} s},-e^{i p_{2} s}\right) \in T^{2} \mid 0 \leqslant s \leqslant 2 \pi\right\} .
\end{aligned}
$$

Hence $K$ is the torus knot of type $\left(\left|p_{1}\right|,\left|p_{2}\right|\right)$.

FutURE DireCtions: In subsequent papers, we shall study the periodicity of symmetric geodesics for the Euclidean Jordan algebra $\operatorname{Sym}(2, \mathbb{R})$ of all $2 \times 2$ symmetric real matrices, realised in the Shilov boundary $\Sigma_{2}$ which is a non-orientable closed 3-manitold. By an investigation of some finite group actions on an orientable double covering space of the Shilov boundary $\Sigma_{2}$, we shall give a certain class of knots and links in $S^{3}$ corresponding to the Euclidean Jordan algebra $\operatorname{Sym}(2, \mathbb{R})$ and will discuss their properties.

\section{APPENDix}

Let $\mathcal{X}(\mathbb{R})$ be the Lie algebra of all smooth vector fields on $\mathbb{R}$. For $g=\left(\begin{array}{ll}a & b \\ c & d\end{array}\right) \in$ $\operatorname{SL}(2, \mathbb{R})$, we consider the linear fractional transformation $g(x)=(a x+b)(c x+d)^{-1}$. This defines a local action on $\mathbb{R}$ and a global action on $S^{1}$. The induced Lie homomorphism $\phi: \mathfrak{s l}(2, \mathbb{R}) \longrightarrow \mathcal{X}(\mathbb{R})$ is given by

$$
\phi\left(\begin{array}{cc}
a & b \\
c & -a
\end{array}\right)=\left(b+2 a x-c x^{2}\right) \frac{\partial}{\partial x} .
$$

In this case, $\mathfrak{q}, \mathfrak{h}$, and $C_{\mathfrak{p}}$ are given by

$$
\begin{aligned}
\mathfrak{q} & =\left\{\left(\begin{array}{ll}
0 & a \\
b & 0
\end{array}\right) \mid a, b \in \mathbb{R}\right\}, \\
C_{\mathfrak{p}} & =\left\{\left(\begin{array}{ll}
0 & a \\
b & 0
\end{array}\right) \mid a, b \geqslant 0\right\}, \\
\mathfrak{h} & =\left\{\left(\begin{array}{cc}
a & 0 \\
0 & -a
\end{array}\right) \mid a \in \mathbb{R}\right\} .
\end{aligned}
$$

It is easy to show that $f\left(\mathfrak{h} \pm C_{\mathfrak{p}}\right)=\infty$. Now let $X=\left(\begin{array}{cc}a & b \\ c & -a\end{array}\right) \in \mathfrak{s l}(2, \mathbb{R})$. Note that $X(x)=b+2 a x-c x^{2}$. Set $\alpha=\left|a^{2}+b c\right|^{1 / 2}$. For $b \neq 0$ and $c \neq 0$, the differential equation 
$x^{\prime}(t)=X(x(t)), x(0)=0$, has the solution:

$$
x(t)= \begin{cases}\frac{a}{c}\left(1+\frac{1}{a t-1}\right) & \text { if } a^{2}+b c=0, \\ \frac{a}{c}+\frac{\alpha}{c} \tanh \left[\alpha t+\tanh ^{-1}\left(\frac{-a}{\alpha}\right)\right] & \text { if } a^{2}+b c>0, b c>0, \\ \frac{a}{c}+\frac{\alpha}{c} \operatorname{coth}\left[\alpha t+\operatorname{coth}^{-1}\left(\frac{-a}{\alpha}\right)\right] & \text { if } a^{2}+b c>0, b c<0, \\ \frac{a}{c}-\frac{\alpha}{c} \tan \left[\alpha t+\tan ^{-1} \frac{a}{\alpha}\right] & \text { if } a^{2}+b c<0 .\end{cases}
$$

Then the function $f$ is given by

$$
f(X)= \begin{cases}\infty & \text { if } X \in \mathfrak{h} \pm C_{\mathfrak{p}}, \\ \infty & \text { if } a^{2}+b c=0, a \leqslant 0, \\ \infty & \text { if } a^{2}+b c>0, b c<0, a<0 \\ a^{-1} & \text { if } a^{2}+b c=0, a>0, \\ \frac{\tanh ^{-1}\left(\frac{\alpha}{a}\right)}{\alpha} & \text { if } a^{2}+b c>0, b c<0, a>0, \\ \frac{\tan ^{-1} \frac{\alpha}{a}}{\alpha} & \text { if } a^{2}+b c<0, a>0, \\ \frac{\pi}{2 \alpha} & \text { if } a^{2}+b c<0, a \leqslant 0 .\end{cases}
$$

\section{REFERENCES}

[1] W. Bertram, 'Un théorème de Liouville pour les algèbres de Jordan', Bull. Soc. Math. France 124 (1996), 299-327.

[2] W. Bertram, 'On some classes and conformal groups', J. Lie Theory 6 (1996), 215-247.

[3] W. Bertram, 'Algebraic structures of Makarevič spaces I', Transform. Groups 1 (1998), 3-32.

[4] J. Faraut and A. Koranyi, Analysis on symmetric cones, Oxford Mathematical Monographs (Oxford University Press, Oxford, 1994).

[5] J. Hilgert and G. Olafsson, Causal symmetric spaces.Geometry and harmonic analysis, Perspectives in Mathematics 18 (Academic Press, San Diego, 1997).

[6] W. Kaup, 'Algebraic characterization of symmetric complex Banach manifolds', Math. Ann. 228 (1977), 39-64.

[7] W. Kaup, 'A Riemann mapping theorem for bounded symmetric domains in complex banach spaces', Math. Z. 183 (1983), 503-529.

[8] K. Koufany, 'Semi-groupe de Lie associé a un cône symétriques', Ann. Inst. Fourier (Grenoble) 45 (1995), 1-29.

[9] J. D. Lawson and Y. Lim, 'Lie semigroups with triple decompositions', (submitted). 
[10] O. Loos, Bounded symmetric domains and Jordan pairs, Lecture Notes (University California, Irvine, 1977).

[11] B. Makarevič, 'Ideal points of semisimple Jordan algebras', Mat. Zametki 15 (1974), 295-305.

[12] D. Rolfsen, Knots and links, Mathematics Lecture Series 7 (Publish or Perișh Press, Berkley, CA, 1976).

Department of Mathematics

College of Natural Sciences

Kyungpook National University

Taegu 702-701

Korea

e-mail: syleek@chollian.net

Department of Mathematics

College of Natural Sciences

Kyungpook National University

Taegu 702-701

Korea

e-mail: chnypark@kyungpook.ac.kr
Topology and Geometry Research Center Kyungpook National University

Taegu 702-701

Korea

e-mail: ylim@math.kyungpook.ac.kr 